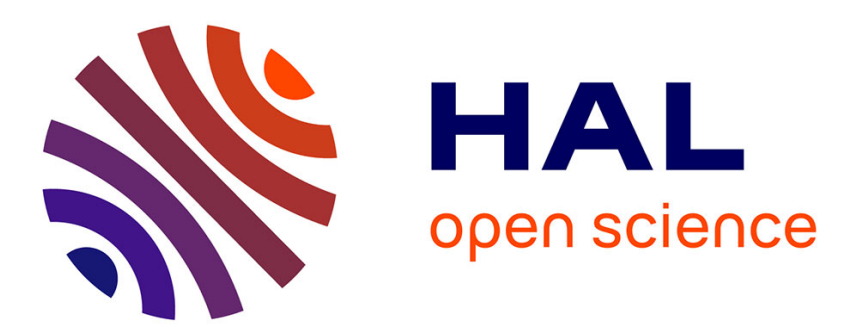

\title{
Proximité spatiale et relations science - industrie : savoirs tacites ou encastrement (Polanyi ou Polanyi)?
}

Michel Grossetti, Marie-Pierre Renée Bes

\section{To cite this version:}

Michel Grossetti, Marie-Pierre Renée Bes. Proximité spatiale et relations science - industrie: savoirs tacites ou encastrement (Polanyi ou Polanyi)?: réflexions à partir d'une étude sur les collaborations CNRS - entreprises en France. Revue d'économie régionale et urbaine, 2003, 5, pp.777-788. halshs00476997

\section{HAL Id: halshs-00476997 https://shs.hal.science/halshs-00476997}

Submitted on 27 Apr 2010

HAL is a multi-disciplinary open access archive for the deposit and dissemination of scientific research documents, whether they are published or not. The documents may come from teaching and research institutions in France or abroad, or from public or private research centers.
L'archive ouverte pluridisciplinaire HAL, est destinée au dépôt et à la diffusion de documents scientifiques de niveau recherche, publiés ou non, émanant des établissements d'enseignement et de recherche français ou étrangers, des laboratoires publics ou privés. 
Michel Grossetti et Marie-Pierre Bès

Centre d'étude des rationalités et des savoirs (Cers)

Université de Toulouse-le-Mirail

5, Allées A. Machado

31058 Toulouse Cedex

Tel : 0561503669

Fax : 0561503870

Email : Michel.Grossetti@univ-tlse2.fr, bes@univ-tlse2.fr

\title{
Proximité spatiale et relations science - industrie : savoirs tacites ou encastrement (Polanyi ou Polanyi)?
}

\author{
réflexions à partir d'une étude sur les collaborations CNRS - entreprises en France
}

Revue d'Economie Régionale et Urbaine, Revue d'Economie Régionale et Urbaine, $\mathrm{n}^{\circ} 5$ II -, pp.777-788, repris dans Problèmes économiques, n².828, 23 Octobre 2003, pp.28-32.

Il était une fois deux frères nés à Budapest, l'un en 1886 (Karl) et l'autre en 1891 (Michael). Karl étudia le droit et l'économie, Michael la médecine et la chimie. Ils durent tous deux, ainsi que d'autres de leurs frères et sœurs émigrer en Grande-Bretagne durant les années trente pour échapper à la menace nazie. Les Polanyi partirent ensuite aux Etats-Unis où ils connurent un certain succès académique. Karl publia en 1944 La grande transformation, un essai sur le capitalisme où il utilise le terme d'encastrement pour indiquer que l'échange marchand de type capitaliste n'a rien de naturel et ne peut se développer que grâce à des institutions juridiques et politiques particulières (Polanyi, 1983). Michael, après de nombreux travaux en médecine et en chimie, se tourna vers la philosophie des sciences. Il publia de nombreux ouvrages dans ce domaine, dont le plus connu est probablement Personal Knowledge (1958), dans lequel il utilise la notion de savoir tacite (développé plus particulièrement encore dans The Tacit dimension, 1962) pour souligner le caractère toujours partiellement incorporé des connaissances scientifiques. Karl est donc pour partie l'inspirateur des théories actuelles de l'encastrement et Michael celui des travaux sur les savoirs tacites. Les deux notions sont cousines, non seulement parce que leurs "pères" respectifs étaient frères, mais parce qu'elles soulignent l'une et l'autre la dimension humaine de processus présentés parfois comme relevant de logiques immanentes. Au fond, la notion d'encastrement souligne la dépendance des échanges économiques vis-à-vis des relations sociales et celle de savoir tacite, la dépendance des connaissances scientifiques vis-à-vis des caractéristiques psychologiques et sociales de ceux qui les construisent.

Les deux notions ont fait l'objet de traductions, de réinterprétations successives, de reformulations diverses et sont devenues des instruments de pensée largement répandus.

L'encastrement a été redéfini par Mark Granovetter comme une dépendance de l'activité économique vis-à-vis des relations personnelles de ceux qui la font exister (Granovetter, 
1985). La notion est alors mise au service d'une thèse différente de celle de Karl Polanyi, pour qui l'autonomie de la sphère économique dans les sociétés industrielles fait contraste avec son intrication dans les relations sociales au sein des sociétés traditionnelles. Pour Granovetter, dans les sociétés industrielles aussi, les activités économiques dépendent fortement des liens sociaux. Cette thèse, reprise depuis par d'autres auteurs (Swedberg, 1997) est à l'origine du courant de la " Nouvelle Sociologie Economique " (Granovetter et Swedberg, 1991).

La notion de savoir tacite a quant à elle été largement reprise par les économistes évolutionnistes, puis par divers auteurs dans le cadre de l'économie de l'innovation. Elle permet d'argumenter la difficulté de réduire l'information scientifique à un bien largement diffusable et reproductible. Ainsi, dans leur théorie évolutionniste du changement économique, Nelson et Winter (1982) se refèrent explicitement à l'ouvrage de Michael Polanyi de 1962 pour l'élaboration de leur théorie de la firme et écrivent à plusieurs reprises que "son influence est forte bien qu'il ne soit pas reconnu comme un théoricien des organisations" (Nelson \& Winter, p.96). Ils reprennent l'idée de "tacit knoweldge", défini comme un ensemble de connaissances non articulées au sein du savoir-faire humain, mais transposé aux capacités organisationnelles des entreprises: "être capable de faire quelque chose et en même temps, être incapable d'expliquer comment on en fait, est plus qu'une possibilité logique, c'est une situation courante" (Nelson \& Winter, p.76) écrivent les deux auteurs. Car les savoirs comportent une part non explicite, soit parce que cela prendrait trop de temps de décrire tout ce qui est nécessaire à leur opérationnalisation, soit parce que c'est intrinsèquement impossible. L'entreprise évolutionniste est alors dotée d'une capacité de mémorisation des compétences humaines, liées aux décisions économiques, appelée "routine". A partir de cet ouvrage, d'autres chercheurs (Dosi, Marengo, Teece, Lazaric) adopteront une vision de la firme comme réceptacle de connaissances explicites et codifiées, existant sous forme de biens marchands, et de connaissances tacites, non transférables d'une entreprise à l'autre.

Utilisées par des chercheurs travaillant sur des objets proches, il fallait bien que les notions cousines en viennent à se rencontrer. La question des effets de proximité dans les relations science-industrie (ou des effets de diffusion localisée de connaissances) est l'un des terrains où cette rencontre s'est effectuée. De quoi s'agit-il ? Les conceptualisations mises en œuvre dans ce domaine sont multiples, mais, pour simplifier, on peut dire que les relations entre les entreprises et les organisations scientifiques sont sensibles à des effets de proximité spatiale, c'est-à-dire que " toutes choses non spatiales que l'on ait pensé à mesurer égales par ailleurs ", les relations entre les laboratoires de recherche publique (ou universitaires, puisque dans le cas américain, de nombreuses universités sont privées) et les entreprises sont plus nombreuses et intenses au sein d'espaces infra-nationaux de l'ordre des bassins d'emploi ou des grandes agglomérations urbaines ${ }^{1}$.

\footnotetext{
${ }^{1}$ Aux Etats-Unis, la question des "geographic localization of knowledge spillovers" revient régulièrement dans les travaux de géographie économique ou d'économie de l'innovation depuis la fin des années quatre-vingt avec les travaux de Acs et Audretsch (1988), Jaffe (1989), Audretsch et Feldman (1994), Mansfield (1994) et bien d'autres. En France, l'analyse des contrats de recherche entre les organismes publics et les entreprises montre l'existence d'effets de proximité aussi bien pour l'Institut National de Recherches Agronomiques (Estades, Joly et Mangematin, 1995) que pour le Centre National de la Recherche Scientifique (Grossetti et Nguyen, 2001). Ces résultats rejoignent les observations plus qualitatives effectuées sur Silicon Valley (Saxenian, 1994) ou d'autres sites comparables.
} 
Pour expliquer ce phénomène les deux notions " polanyiennes" sont mises au service de deux thèses qui émergent particulièrement dans les travaux récents.

L'encastrement est mis en avant dans des travaux directement inspirés de la nouvelle sociologie économique comme l'étude de Powell et Brantley sur les biotechnologies en Californie (Powell et Brantley, 1992) ou qui s'y réfèrent explicitement comme ceux de Saxenian sur Silicon Valley (Saxenian, 1994) ou Grossetti sur Toulouse (Grossetti, 1995). Le terme d" encastrement" est en général utilisé dans ces travaux pour désigner le fait que les échanges entre entreprises et laboratoires dépendent des réseaux personnels des membres de ces organisations. Ces réseaux étant en grande partie locaux, cela explique les effets de proximité dans les échanges entre organisations. Sans forcément utiliser le terme d'encastrement, d'autres travaux insistent sur l'effet des réseaux sociaux. Ainsi Joly, Lemarié et Mangematin (1995), dans une étude des relations entre les entreprises et les laboratoires de l'INRA concluent à l'existence de trois types de logiques dont la logique de "proximité" dans lesquelles le milieu local et les réseaux d'interconnaissance jouent un rôle central. Une autre étude réalisée dans la lignée de ce premier travail sur les relations entre des équipes du CEA et de l'INRA et des petites entreprises montre un effet important des relations interpersonnelles préalables (Genet, 1999). La critique que l'on fait parfois à ces travaux est de surestimer le poids des relations sociales et d'oublier l'importance des dispositifs institutionnels qui structurent les marchés.

La seconde thèse insiste sur le caractère tacite donc peu transportable des savoirs nouveaux, qui impliquent donc des échanges en face à face favorisant les relations de proximité. Ainsi, selon Dominique Foray (1995) : "le facteur géographique est important (...) des agglomérations industrielles, situées en un lieu plutôt qu'en un autre, créent un environnement dans lequel l'expérience de production peut être accumulée, échangée et conservée par la main d'oeuvre et la communauté d'entreprises locales. De même, l'aptitude à assimiler et à transférer les connaissances scientifiques et technologiques qui ne sont pas totalement codifiées est largement tributaire des opportunités de contact personnel direct entre les parties concernées." (Foray, 1995, p. 19). Pour Zucker, Darby et Armstrong (1994), "Les effets géographiquement localisés se produisent pour les découvertes scientifiques caractérisées par une exclusivité naturelle, celles qui ne peuvent être apprises qu'en travaillant avec les découvreurs ou avec ceux qui ont acquis les connaissances nécessaires en travaillant avec eux dans le laboratoire." (1994, résumé). Les auteurs ont étudié 110 nouvelles entreprises de biotechnologie de Californie, la plupart fondées explicitement pour exploiter les résultats obtenus par certains chercheurs, les chercheurs en question étant souvent impliqués directement dans la nouvelle entreprise. Utilisant des données statistiques sur les entreprises et des données bibliométriques sur les publications réalisées par des membres des entreprises avec des chercheurs, les auteurs trouvent que " pour toutes les mesures de performance de la firme, la présence de $\operatorname{stars}^{2}$ liées $^{3}$ à l'entreprise a un effet positif significatif alors que la présence d'autres stars universitaires locales et de stars affiliées à la firme ${ }^{4} n^{\prime} e n$ ont pas lorsque les trois variables sont prises en compte dans une même équation" (p.24). Autrement dit lorsqu'un chercheur travaille à la fois dans son laboratoire et dans la firme, celle-ci est plus performante. Peut-on en conclure pour autant que "les effets de diffusion

\footnotetext{
2 Les stars sont les chercheurs les plus connus en termes de publications et de citations.

${ }^{3}$ ayant publié avec des membres de l'entreprise ou signé certaines publications en indiquant l'entreprise comme affiliation.

${ }^{4}$ Signant tous leurs articles en indiquant la firme comme employeur ou affiliation.
} 
géographiquement localisés de la connaissance représentent en fait un échange marchand spécifique " (p.25) ? Rien n'est moins sûr. Après tout, le fait que les entreprises créées par (ou en relation forte avec) des chercheurs continuant à travailler à l'université soient à proximité de celle-ci peut s'expliquer par des raisons aussi triviales que la volonté des chercheurs en question d'éviter de disperser leurs lieux de travail et non par les caractéristiques intrinsèques des connaissances produites. Cela peut aussi s'expliquer par la plus grande disponibilité dans le milieu local de ressources (capitaux, force de travail, etc.) pour créer de nouvelles entreprises. Mais surtout on observe des effets de proximité dans de nombreux cas où les chercheurs n'ont pas d'intérêt particulier dans les firmes, ou encore lorsqu'il s'agit de grandes firmes ayant de multiples établissements dont la localisation est largement antérieure à l'établissement des collaborations ou encore lorsqu'il n'y a pas d'activité de recherche et développement (voir par exemple la diffusion des innovations concernant la plasturgie à Oyonnax dans Raveyre et Saglio, 1984).

C'est entre autres pour essayer de tester en quelque sorte ces deux thèses que nous avons reconstruit 130 histoires de collaborations entre des laboratoires du CNRS et des industriels ${ }^{5}$. Nous avons dans un autre cadre détaillé les résultats de cette étude à l'appui d'une conception spécifique des notions d'encastrement et de découplage (Grossetti et Bès, 2001). Nous ne reprenons ici que les éléments qui peuvent éclairer la question des effets de proximité dans les relations science-industrie et la place que l'on peut faire aux thèses de l'encastrement ou des savoirs tacites.

\section{Les savoirs tacites n'expliquent pas les effets de proximité spatiale}

Rappelons que l'explication des effets de proximité par les contraintes d'échange des savoirs tacites, retenue par plusieurs auteurs, postule que les membres des entreprises doivent pouvoir se rendre dans le laboratoire pour accéder aux savoirs tacites des chercheurs, ce qui favorise les coopérations locales. Nous prenions cette hypothèse très au sérieux en commençant l'enquête et nous avons posé des questions systématiques sur le rythme des réunions, les visites réciproques dans l'entreprise ou le laboratoire, les problèmes ou difficultés de compréhension. Nous pensons à présent que cette hypothèse est totalement erronée, au moins dans le cas que nous avons étudié.

En effet, nous n'avons jamais rencontré de cas où les ingénieurs ou membres des entreprises se soient rendus régulièrement dans les laboratoires partenaires en dehors des 3 ou 4 réunions annuelles de coordination du projet, rythme qui ne varie pratiquement pas et ne différencie en rien les relations locales des autres. Lorsqu'une coopération nécessite des échanges très importants, ce qui est fréquent mais pas systématique, il y a

\footnotetext{
5 Nous avons réalisé 27 entretiens initiaux avec des chercheurs, qui ont généré 130 histoires, que nous avons complétées par 119 entretiens (73 avec les partenaires industriels, 46 avec d'autres chercheurs ou participants). Les chercheurs interrogés initialement sont dans des équipes de Toulouse (11), de Bordeaux (5), de Montpellier (3), de Clermont-Ferrand (2) et de Grenoble (6). La plupart des spécialités des SPI sont représentées : électrotechnique (1), électronique (7), automatique ou robotique (3), informatique (5), génie des procédés (4), mécanique des fluides (7). Les 130 relations concernent 81 partenaires dont 38 groupes industriels ( 73 relations), 35 petites entreprises (38 relations) ${ }^{5}$ et 8 organismes publics de recherche technologique (CNES, CNET, etc.) (19 relations), que nous avons décidé d'inclure parce qu'ils ont joué véritablement un rôle de partenaire industriel dans les histoires recueillies. $36 \%$ de ces relations sont locales (laboratoire et entreprise dans la même région), $38 \%$ associent un laboratoire de province et une entreprise de la région parisienne et $26 \%$ un laboratoire et un partenaire d'une autre région ou d'un autre pays. Voir (Grossetti et Bès, 2001) pour une description plus détaillée de la méthode et des résultats.
} 
généralement un doctorant qui fait des séjours successifs plus ou moins longs dans le laboratoire et dans l'entreprise. Les contraintes techniques que peut impliquer le travail dans deux sites éloignés est pallié sans difficulté par des méthodes comme le double maquettage (en électrotechnique par exemple), où une maquette identique du système à étudier est réalisée simultanément au laboratoire et dans l'entreprise. L'idée que les membres de l'entreprise cherchent à s'approprier les savoirs des chercheurs, tacites ou non, ne résiste pas non plus à l'analyse. Ce qui intéresse les entreprises interrogées c'est le résultat, et non pas nécessairement les méthodes ou les concepts mais si c'est le cas, le recrutement d'un doctorant règle cette question.

Cela ne signifie pas pour autant qu'il n'y a pas de dimension tacite dans ces échanges. Les savoirs tacites se situent à quatre niveaux différents. Le premier concerne la compréhension par les industriels de ce que c'est que la recherche et les chercheurs. Cette compréhension, aisée lorsqu'il s'agit de grands groupes et de leurs centres de recherche peuplés d'ingénieurs dont certains ont fait des thèses, ou de petites entreprises de haute technologie, elles aussi peuplées d'ingénieurs et de docteurs, devient nettement plus problématique avec des responsables de petites entreprises traditionnelles qui tendent à formuler des problèmes décalés par rapport à ce qu'attendent ou peuvent faire les chercheurs. D'où de nombreuses difficultés dans la définition des problèmes à traiter et dans l'évaluation des efforts. Plusieurs chercheurs ont cité des cas où les responsables de petites entreprises attendaient en six mois un résultat qui demandait selon eux plusieurs années de travail ou ne percevaient pas l'importance de définir des sujets de thèse suffisamment larges pour être validés sur le plan académique. Le second niveau de savoir tacite concerne les disciplines de formation des personnes amenées à collaborer. Par exemple, il est plus facile de se comprendre entre chimistes, même si l'un est dans une entreprise et l'autre dans un laboratoire, qu'entre un chimiste et un électronicien. Il y a aussi le niveau du métier de l'entreprise et des implications pour elle du problème posé, que les chercheurs mettent toujours un certain temps à appréhender. Enfin, les savoir-faire des chercheurs constituent un quatrième niveau dont nous avons vu qu'il ne débouche pas sur des contraintes de proximité.

Il existe donc bien des savoirs tacites, multiples, mais il n'expliquent en rien les effets de proximité spatiale. Le travail en collaboration n'est pas particulièrement plus difficile à distance à condition que certains participants puissent se déplacer sans problème et que les dispositifs techniques et organisationnels soient adaptés à cette situation.

\section{Les effets d'encastrement dans la genèse des collaborations}

Notre analyse des logiques de construction des collaborations retrouve en partie la typologie présentée dans l'étude conduite sur les laboratoires de l'INRA (Estades, Joly et Mangematin, 1996). Nous avons aussi regroupé les situations rencontrées en trois catégories, mais celles-ci ne tiennent compte ni du caractère local ou non des collaborations, ni de l'origine de l'initiative. Elles sont centrées sur le contexte du contact. Dans la première catégorie - la logique de réseau - le contact résulte de l'existence d'une chaîne relationnelle préalable reliant les personnes décidant de la collaboration. Dans la deuxième catégorie - la logique d'institution — le contact est établi sous l'égide d'une instance extérieure qui provoque, volontairement ou non, les interactions entre membres des organisations qui seront amenées par la suite à collaborer. Enfin dans la troisième catégorie — la logique de " marché" — le contact résulte de l'initiative d'un 
des partenaires (sur la base des informations publiques comme par exemple les publications scientifiques) ou de leur rencontre dans une manifestation collective (congrès, salon).

Lorsque l'on croise ces logiques de rencontre et la localisation des partenaires, on obtient des résultats qui plaident clairement pour l'explication des effets de proximité par l'existence de réseaux personnels locaux.

\section{Type de contact et localisation des partenaires}

\begin{tabular}{|c|c|c|c|c|}
\hline $\begin{array}{l}\text { Type de contact } \\
\text { Localisation des partenaires }\end{array}$ & Réseaux & Institutions & Marché & Total \\
\hline $\begin{array}{l}\text { Etablissement industriel partenaire dans } \\
\text { la même région que le laboratoire }\end{array}$ & $\begin{array}{c}24 \\
(60 \%)\end{array}$ & $\begin{array}{c}8 \\
(20 \%)\end{array}$ & $\begin{array}{c}8 \\
(20 \%)\end{array}$ & $\begin{array}{c}40 \\
(36 \%)\end{array}$ \\
\hline $\begin{array}{l}\text { Etablissement industriel partenaire à } \\
\text { Paris }\end{array}$ & $\begin{array}{c}17 \\
(41 \%)\end{array}$ & $\begin{array}{c}7 \\
(17 \%)\end{array}$ & $\begin{array}{c}17 \\
(42 \%)\end{array}$ & $\begin{array}{c}41 \\
(37 \%)\end{array}$ \\
\hline $\begin{array}{l}\text { Etablissement industriel partenaire dans } \\
\text { une autre région ou à l'étranger }\end{array}$ & $\begin{array}{c}7 \\
(24 \%)\end{array}$ & $\begin{array}{c}5 \\
(17 \%)\end{array}$ & $\begin{array}{c}17 \\
(59 \%)\end{array}$ & $\begin{array}{c}29 \\
(27 \%)\end{array}$ \\
\hline Total & $\begin{array}{c}48 \\
(44 \%)\end{array}$ & $\begin{array}{c}20 \\
(18 \%\end{array}$ & $\begin{array}{c}42 \\
(38 \%)\end{array}$ & $\begin{array}{c}110 \\
100 \%\end{array}$ \\
\hline
\end{tabular}

$($ Khi2 $=11,89, \mathrm{p}=0,018)$

Même si la corrélation est nette, il faut bien se garder de confondre les relations locales et la logique des réseaux puisque celle-ci s'applique largement à des cas où les partenaires sont éloignés. Les relations décisives (celles qui font le pont entre les organisations) impliquées dans les contacts par réseaux sont issues quatre fois sur cinq de l'univers de l'enseignement (anciens élèves, professeur/élève) ou de l'activité professionnelle (collègues). Les relations science-industrie sont donc dépendantes pour partie d'autres types d'échanges que sont les activités d'enseignement des chercheurs et le marché du travail.

Il y a toutefois une limite à l'encastrement des relations entre organisations dans les réseaux personnels. Dans nos données, les logiques de contact entre les entreprises et les laboratoires n'apparaissent corrélées ni avec la localisation des laboratoires étudiés, ni avec le type de partenaire, ni avec les contenus technologiques, ni avec la durée des collaborations. Tout se passe comme si la localisation géographique des partenaires était la principale conséquence du contexte de formation de la collaboration sur le déroulement de celle-ci. L'oubli des conditions de construction de la relation passe par des processus qui peuvent produire du " découplage ", c'est-à-dire un affaiblissement de la dépendance des relations entre organisations vis-à-vis des relations personnelles ou au contraire un renforcement de cet encastrement. Ces processus bien connus vont du cadre juridique formel que constitue le contrat aux dispositifs techniques permettant le travail en commun (maquettage, modélisation, logiciel spécifique, instruments, données produites, matériaux, documents intermédiaires) en passant par les règles internes des organisations. Nous avons insisté dans notre analyse sur un autre processus, la "personnification", qui se fonde sur l'implication d'acteurs sociaux particuliers, qui incarnent pour un temps la relation : stagiaires, doctorants co-financés, chercheurs ou ingénieurs détachés. Ces acteurs appartiennent pour un temps aux deux collectifs. On 
pourrait dire qu'il appartiennent au collectif de la collaboration et du projet qui lui est associé. Dans plus de neuf cas sur dix, la collaboration repose sur le travail de ces acteurs intermédiaires. Comme les intermédiaires matériels, les intermédiaires humains contribuent à rendre la relation partiellement autonome des collectifs impliqués. En même temps, dans la mesure où les thésards ou les stagiaires tendent à se faire recruter par les entreprises participantes, et où les ingénieurs ou les chercheurs conservent des relations avec leurs anciens collègues, ce quatrième processus produit de l'encastrement dans les réseaux sociaux et peut contribuer à défaire les savants découplages issus des trois précédents.

\section{Karl ou Michael ?}

Notre étude montre que les collaborations entre entreprises et laboratoires sont bien, pour une part importante, encastrées dans des réseaux sociaux. Mais cet effet d'encastrement s'exerce de façon temporaire, au moment où la relation se construit, et peut lui-même s'expliquer par l'existence de sphères d'échange connexes aux relations entre entreprises et laboratoires, en particulier l'enseignement et les marchés du travail. Les logiques d'encastrement, qui peuvent s'exercer aussi après le début de la collaboration, quelle que soit l'origine de celle-ci, sont toujours en tension avec des processus de découplage que nous avons décrits plus haut. La résultante de ces différents processus peut aboutir à des situations très variables selon les collaborations, mais les régularités que nous avons mises en évidence en ce qui concerne les contacts entre les partenaires permettent entre autres d'expliquer les effets de proximité spatiale. Dans les cas que nous avons étudiés, ces effets sont dus pour l'essentiel à l'influence de réseaux sociaux - qui sont en partie locaux - au moment de la construction des collaborations, et non par des contraintes d'échanges de savoirs tacites. C'est Karl qu'il faut aller chercher pour expliquer les effets de proximité.

\section{Références}

AUDRETSCH David B. et FELDMAN Maryann P. (1996), "R\&D spillovers and the geography of innovation and production", The American Economic Review, vol 86, n 3, Juin.

CALLON Michel, 1999, "Une contribution de la sociologie à l'analyse des externalités. Essai sur la notion de cadrage/débordement", in D. Foray et J. Mairesse, Innovation et performance, EHESS.

CASSIER Maurice, 1997, "compromis institutionnels et hybridations entre recherche publique et recherche privée", Revue d'économie industrielle, $\mathrm{n}^{\circ} 79$, p. 191-212.

CASSIER Maurice, 1999, "Le partage des connaissances dans les réseaux scientifiques : l'invention des règles de "bonne conduite" par les chercheurs", Revue Française de Sociologie, pp.701-720

ESTADES Jaqueline, JOLY Pierre-Benoit et MANGEMATIN Vincent, 1996, "Dynamique des relations industrielles dans les laboratoires d'un grand organisme public de recherche : coordination, apprentissage, réputation et confiance", Sociologie du Travail, n³, p.391-407.

FORAY Dominique, 1995 : "distribution et expansion de la base de connaissances scientifiques et technologiques", revue STI, OCDE, pp.14-71.

GENET Corine, 1999, "Dynamique des cooperations entre la recherche publique et les pme : quelle est la place des relations informelles ? Etude de cas à partir des laboratoires du CEA et de l'INRA", Colloque ADIS, "Coopération Industrielle : Diversité et Synthèse", 3 et 4 Mai 1999

GRANOVETTER Mark et SWEDBERG Richard (eds), 1991, The sociology of economic life, Westview Press, Boulder, San Francisco, Oxford. 
GRANOVETTER Mark, 1985, "Economic action and social structure : the problem of embeddedness", American Journal of Sociology, Vol. 91, n³, pp.481-510.

GRANOVETTER Mark, 1994, "Les institutions économiques comme constructions sociales : un cadre d'analyse", in A. Orléan (ed), L'analyse économique des conventions, PUF, pp.79-94.

GROSSETTI Michel et BES Marie-Pierre, 2001, "Encastrements et découplages dans les relations science - industrie ", Revue Française de Sociologie, Vol. 42, n², pp.327-355.

GROSSETTI Michel et NGUYEN David, 2001, "La structure spatiale des relations science-industrie en France : l'exemple des contrats entre les entreprises et les laboratoires du CNRS ", Revue d'Economie Régionale et Urbaine, II, pp.311-328.

GROSSETTI Michel, 1995, Science, industrie et territoire, Presses Universitaires du Mirail, Coll. Socio-logiques.

GROSSETTI Michel, 1998, "La proximité en sociologie : une réflexion à partir des systèmes locaux d'innovation", in BELLET Michel, KIRAT Thierry et LARGERON Christine (dir.), 1998, Approches mutiformes de la proximité, Coll. "Interdisciplinarité et nouveaux outils", Hermès, Paris, pp.83-101.

GROSSETTI Michel, 2001, "Les effets de proximité spatiale dans les relations entre organisations : une question d'encastrements", "Espaces \& Sociétés", n¹01-102, pp.203-219

JAFFE Adam B. (1989), " Real effects of academic research ", The American Economic Review, vol. 79, ${ }^{\circ}$ 5, décembre, pp.957-970

KIRMAN Alan et ZIMMERMANN Jean-Benoît, 2001, Economics with heterogeneous interacting agents, Springer.

NELSON R.R.\& WINTER S.G., 1982 : An Evolutionary Theory of Economic Change, Harvard University Press, Cambridge, MA.

POLANYI Karl, 1983, La grande transformation. Aux origines politiques et économiques de notre temps, Gallimard, Paris.

POLANYI Michael, 1958, Personal Knowledge. Towards a Post-Critical Philosophy. (Gifford Lectures, University of Aberdeen, 1951-52). London: Routledge \& Kegan Paul: Chicago: Chicago University Press POLANYI Michael, 1962, The Tacit Dimension (Terry Lectures, Yale University, 1962). Garden City: Doubleday 1966; London: Routledge and Kegan Paul 1967, 108p.; repr. Gloucester, Mass.: Peter Smith 1983;

POWELL Walter S. et BRANTLEY Peter, 1992, "Competitive cooperation in biotechnology : learning through networks ?", in N. Nohria et R.G. Eccles, Networks and organizations : structure, form and action, Boston, Harvard Business School Press, pp.366-394.

POWELL Walter W. and SMITH-DOERR L., 1994, "Networks and économic life", in Neil J. Smelser and Richard Swedberg (eds), The Handbook of economic sociology, Princeton, Russel Sage Foundation.

SAXENIAN AnnaLee, 1994, Regional advantage : culture and competition in Silicon Valley and Route 128, Cambridge (Mas), Harvard University Press.

WHITE Harrison C., 1992, Identity and control. A structural theory of action, Princeton University Press, Pinceton.

ZUCKER Lynne G., DARBY Michael R. et ARMSTRONG Jeff, 1994, " Intellectual capital and the firm : the technology of geographically localized knowledge spillovers ", NBER Working Paper Series, Working Paper n4946, NBER, Cambridge, Mass. 